\title{
RadioAstron Early Science Program Space-VLBI AGN survey: strategy and first results
}

\author{
Kirill V. Sokolovsky* \\ Astro Space Center, Lebedev Physical Inst. RAS, Profsoyuznaya 84/32, 117997 Moscow, Russia \\ Sternberg Astronomical Institute, Moscow University, Universitetsky 13, 119991 Moscow, Russia \\ E-mail: kirx@scan.sai.msu.ru
}

\section{for the RadioAstron AGN Early Science Working Group}

RadioAstron is a project to use the $10 \mathrm{~m}$ antenna on board the dedicated SPEKTR-R spacecraft, launched on 2011 July 18, to perform Very Long Baseline Interferometry from space - SpaceVLBI. We describe the strategy and highlight the first results of a 92/18/6/1.35 cm fringe survey of some of the brighter radio-loud Active Galactic Nuclei (AGN) at baselines up to 25 Earth diameters $\left(D_{\oplus}\right)$. The survey goals include a search for extreme brightness temperatures to resolve the Doppler factor crisis and to constrain possible mechanisms of AGN radio emission, studying the observed size distribution of the most compact features in AGN radio jets (with implications for their intrinsic structure and the properties of the scattering interstellar medium in our Galaxy) and selecting promising objects for detailed follow-up observations, including Space-VLBI imaging. Our survey target selection is based on the results of correlated visibility measurements at the longest ground-ground baselines from previous VLBI surveys. The current long-baseline fringe detections with RadioAstron include OJ 287 at $10 D_{\oplus}(18 \mathrm{~cm})$, BL Lac at $10 D_{\oplus}(6 \mathrm{~cm})$ and B0748+126 at $4.3 D_{\oplus}(1.3 \mathrm{~cm})$. The 18 and $6 \mathrm{~cm}$-band fringe detections at $10 D_{\oplus}$ imply brightness temperatures of $T_{b} \sim 10^{13} \mathrm{~K}$, about two orders of magnitude above the equipartition inverse Compton limit. These high values of $T_{b}$ might indicate that the jet flow speed is often higher than the jet pattern speed.

11 th European VLBI Network Symposium \& Users Meeting,

October 9-12, 2012

Bordeaux, France

* Speaker. 


\section{The space radio telescope}

The $10 \mathrm{~m}$ space radio telescope of the RadioAstron project is installed on board of the dedicated SPEKTR-R spacecraft. The spacecraft was launched into a highly elliptical orbit on 2011 July 18 from the Baikonur Cosmodrome by a Zenit-3F rocket (Figure 1). The orbit was selected so that its parameters evolve under the gravitational pull of the Moon, to provide a wide range of projected baselines for VLBI observations of various sky regions during the mission lifespan. As of 2012 October 2, the SPEKTR-R orbital parameters were: 206-hour period, $73000 \mathrm{~km}$ perigee, $281000 \mathrm{~km}$ apogee, and $79^{\circ}$ inclination. Regular measurements of the spacecraft's distance and velocity using standard radiometric techniques supported by laser ranging, direct optical imaging and VLBI state vector measurements [1] allow one to reconstruct its position and velocity with accuracies typically better than $\pm 500 \mathrm{~m}$ and $\pm 2 \mathrm{~cm} \mathrm{~s}^{-1}$, respectively.

The space telescope is equipped with $92 \mathrm{~cm}$ (324 MHz, P-band), $18 \mathrm{~cm}$ (1.7,GHz, L-band), $6 \mathrm{~cm}(4.8 \mathrm{GHz}, \mathrm{C}$-band) and $1.3 \mathrm{~cm}(22.2 \mathrm{GHz}, \mathrm{K}$-band) receivers, an on-board hydrogen maser, and a high-gain antenna system to downlink VLBI data to a ground station in real time. Currently, the $22 \mathrm{~m}$ antenna of the Pushchino radio astronomy observatory near Moscow, Russia serves as the ground data acquisition station. The second RadioAstron data acquisition station is under construction on the basis of the National Radio Astronomy Observatory's $43 \mathrm{~m}$ telescope at Green Bank, West Virginia, USA.
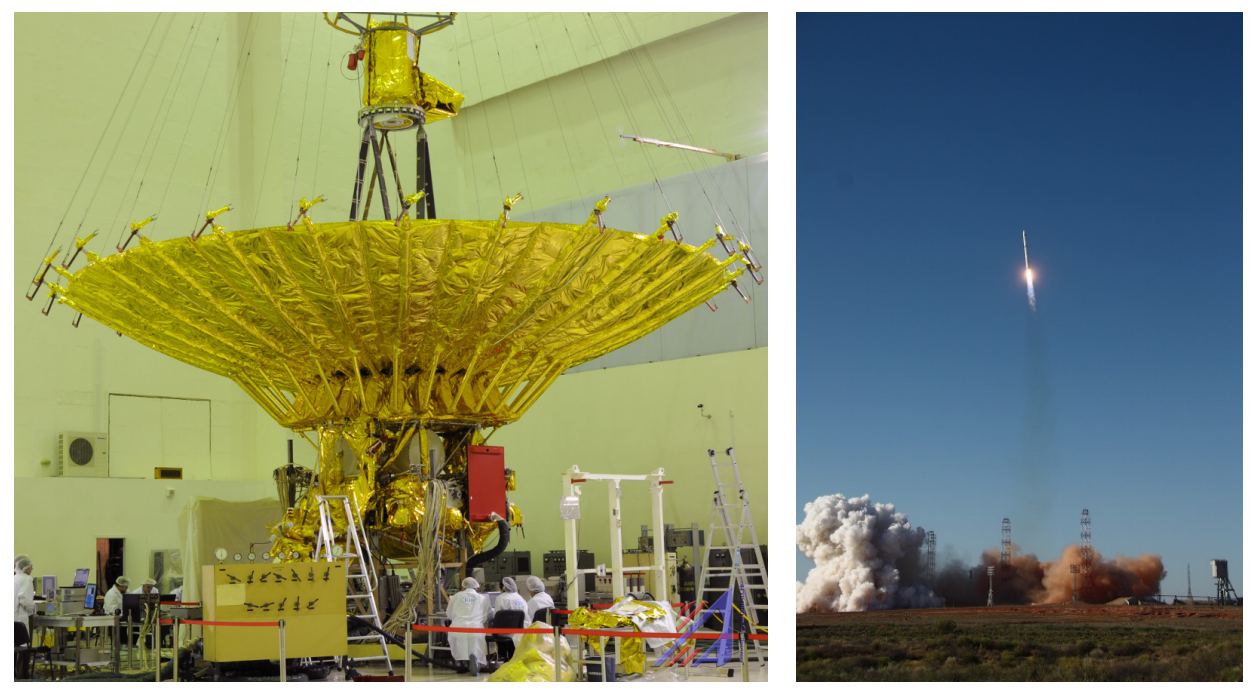

Figure 1: SPEKTR-R assembled at Lavochkin Association (left) and its launch from Baikonur (right).

\section{AGN survey strategy}

A fringe detection survey of radio-bright AGN is being conducted as part of the RadioAstron Early Science Program. The survey goals include a search for extreme brightness temperatures, $T_{b}$, to resolve the Doppler crisis [2] and to constrain possible mechanisms of AGN radio emission [3], to study the size distribution of the most compact features in AGN radio jets (with implications for their intrinsic structure and the properties of the scattering in the interstellar medium in our Galaxy) and select promising targets for detailed follow-up observations, including space-VLBI imaging. 
The survey target selection is based on the results of the correlated visibility measurements on the longest ground-ground baselines from existing VLBI surveys including the $13 / 3.6 \mathrm{~cm}$ (S/Xband) VLBA Calibrator Surveys (VCS) 1 to $6[4,5,6,7,8,9]$ and the Research and Development VLBA program (RDV) $[10,11,12,13], 2 \mathrm{~cm}\left(\mathrm{~K}_{\mathrm{u}}\right.$-band) observations of the MOJAVE program ${ }^{1}$, $7 \mathrm{~mm}$ (Q-) and $3 \mathrm{~mm}$ (W-band) results of the Boston University group ${ }^{2}$ and of a Global $86 \mathrm{GHz}$ VLBI Survey of Compact Radio Sources [14], respectively. We also consulted the list of high- $T_{b}$ sources observed at $6 \mathrm{~cm}$ by the VLBI Space Observatory Programme (VSOP) [15, 16, 17].

The results of many ground-based VLBI surveys are summarized in the Radio Fundamental Catalog ${ }^{3}$. We used the "unresolved X-band flux density" parameter listed in this catalog to set scheduling priorities for the sources. Among sources with comparable unresolved flux densities that satisfy RadioAstron visibility constraints, preference is given to sources for which $(i)$ the correlated visibility at $13,3.6,2 \mathrm{~cm}$ and $7 \mathrm{~mm}$ bands measured from the ground is not decreasing rapidly with increasing baseline length, (ii) we can obtain both short $\left(<5 D_{\oplus}\right)$ and long projected space-ground baselines within one or a few SPEKTR-R orbital revolutions, (iii) VSOP measured $T_{b}>10^{12} \mathrm{~K}$, and (iv) there is a $3 \mathrm{~mm}$ detection. We try to schedule the preferred sources first to maximize the detection rate in the early stages of the survey.

All four RadioAstron bands (92, 18, 6, and $1.3 \mathrm{~cm}$ ) are employed in the survey, with the main focus on 18,6 , and $1.3 \mathrm{~cm}$ bands. Most observations are done in a dual-band mode: $6+18 \mathrm{~cm}$ or $6+1.3 \mathrm{~cm}$. The space radio telescope observes simultaneously at two bands while the ground telescopes are divided in two sub-arrays or switch between the two bands during the experiment. A typical AGN survey observation consists of four scans, each 10-minute long, on a target source, separated from other series of scans by the 40-60 minutes necessary to satisfy the spacecraft's thermal constraints and slew to the next target. The main factors affecting the scheduling include: a Sun avoidance angle of $90^{\circ}$ plus a small solid angle centered on anti-solar direction, satellite visibility to

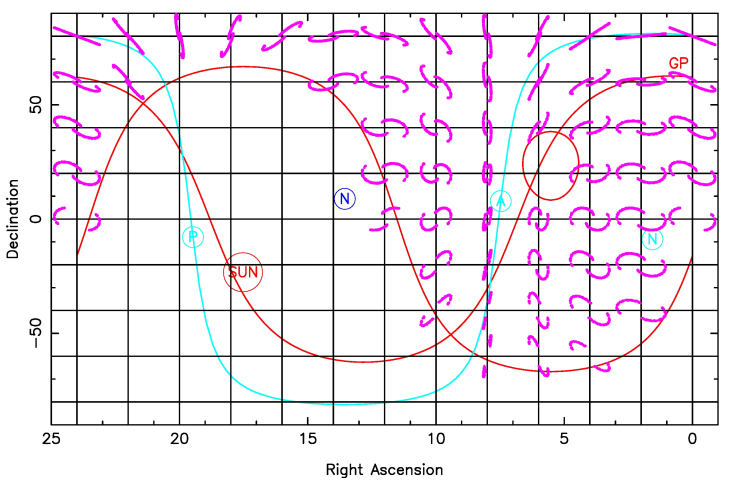

Figure 2: Possible space-ground $(u, v)$-coverage for various sky positions computed for 206 hours, one orbital revolution, starting on 2012 December 15 00:00 UT with the telescopes: RadioAstron, EVN, LBA, Arecibo, GBT, Usuda. Also marked on the plot are: the Galactic Plane (GP), Sun avoidance regions, the satellite's orbital plane, the perigee $(\mathrm{P})$ apogee $(\mathrm{A})$, and the direction perpendicular to the orbital plane. the tracking station (TS), TS visibility to the satellite's high-gain antenna, target source visibility for ground telescopes, and availability of ground telescopes during the time period when all the other constraints are met. An example of the $(u, v)$-coverage computed with the above constraints, except the last one, over the whole sky is presented on Figure 2, although it should be noted that the plot represents a period of rather

\footnotetext{
${ }^{1}$ http://www.physics.purdue.edu/astro/MOJAVE/

${ }^{2}$ http: //www.bu.edu/blazars/

${ }^{3}$ http: //astrogeo.org/rfc
} 
favorable observing conditions.

Ground telescopes participating in the AGN Early Science Program observations include the Arecibo $300 \mathrm{~m}$ and NRAO GBT $100 \mathrm{~m}$ (USA), ATCA tied-array of 5x22 m, Parkes $64 \mathrm{~m}$, Mopra $22 \mathrm{~m}$, Hobart $26 \mathrm{~m}$, Tidbinbilla $70 \mathrm{~m}$ (Australia), Effelsberg $100 \mathrm{~m}$ (Germany), Evpatoria $70 \mathrm{~m}$ (Ukraine), Hartebeesthoek $26 \mathrm{~m}$ (South Africa), Jodrell Bank $70 \mathrm{~m}$ (UK), Medicina $32 \mathrm{~m}$ and Noto $32 \mathrm{~m}$ (Italy), Shanghai $25 \mathrm{~m}$ and Urumqi $25 \mathrm{~m}$ (China), Svetloe $32 \mathrm{~m}$, Zelenchukskaya $32 \mathrm{~m}$, Badary $32 \mathrm{~m}$ (Russia), Torun $32 \mathrm{~m}$ (Poland), Usuda $64 \mathrm{~m}$ (Japan), WSRT 14x25 m (Netherlands), Yebes $40 \mathrm{~m}$ and Robledo $70 \mathrm{~m}$ (Spain), as well as the EVN, Kvazar-KVO, and LBA arrays.

\section{First results}

While the first months of the survey were marked by continuing development of correlation techniques, choosing optimal space telescope observing modes, and debugging the satellite VLBI data downlink system, the observations during this period nonetheless provided some record-breaking results. Fringes to the space telescope at 18 and $6 \mathrm{~cm}$ bands were detected on projected baselines of about $10 D_{\oplus}$ for blazars B0748+126, OJ 287, and BL Lacertae. The ground array consisted of Arecibo, GBT, and Effelsberg telescopes. At $1.3 \mathrm{~cm}$, fringes between the space telescope and the GBT were detected for $\mathrm{B} 0748+126$ at projected baselines up to $4.3 D_{\oplus}$ (Fig. 3). Some of the current long-baseline fringe detections are pre-

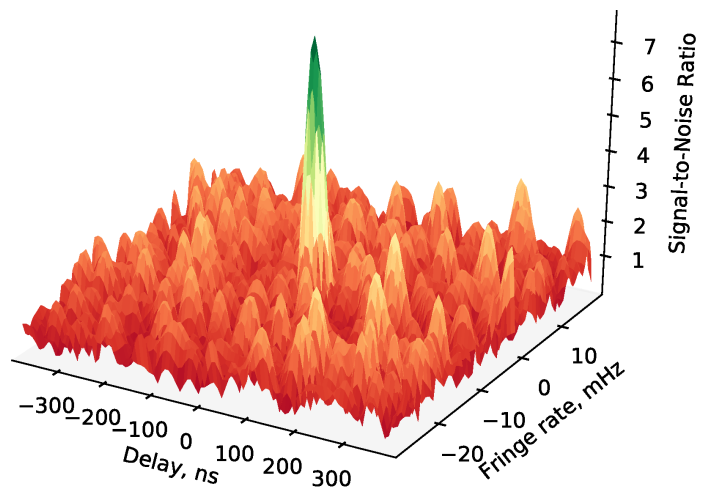

Figure 3: Interferometric signal from the quasar B0748 + 126 detected between RadioAstron and GBT $(1.3 \mathrm{~cm})$ at the projected baseline of $4.3 D_{\oplus}$. The plot shows the signal to noise ratio as a function of residual delay and rate after delay-model subtraction and fringe fitting. sented in Table 1. Both B0748+126 and OJ 287 exhibit interstellar scintillation as determined in the MASIV Survey [18]; BL Lac was not included in MASIV.

While the $92 \mathrm{~cm}$ band is actively used for RadioAstron observations of pulsars, no $92 \mathrm{~cm}$ space-ground fringes on AGN have been detected (few attempts so far). This band was given a low priority because interstellar scattering is most prominent at longer wavelengths and the angular resolution is lower than that of other RadioAstron bands. However, detection of $18 \mathrm{~cm}$ fringes at $10 D_{\oplus}$ suggests that interstellar scattering might not always prevent low-frequency fringe detections at long baselines and more $92 \mathrm{~cm}$ AGN observations should be attempted.

\section{Summary}

The RadioAstron space interferometer is exploring angular scales that were never before accessible at centimeter wavelengths. The 18 and $6 \mathrm{~cm}$ fringe detections at $10 D_{\oplus}$ imply brightness temperatures $T_{b} \sim 10^{13} \mathrm{~K}$, about two orders of magnitude above the equipartition inverse Compton 
Table 1: Some of RadioAstron long-baseline detections as of January 2013

\begin{tabular}{|c|c|c|c|c|c|c|}
\hline Name & Alias & $\begin{array}{l}\text { Ground } \\
\text { telescope }\end{array}$ & $\begin{array}{l}B_{\max } \\
\left(D_{\oplus}\right)\end{array}$ & $\begin{array}{c}B_{\max } / \lambda \\
(\mathrm{M} \lambda)\end{array}$ & $\begin{array}{c}\lambda / B_{\max } \\
(\mathrm{mas})\end{array}$ & $z$ \\
\hline & & \multicolumn{5}{|c|}{ L-band $(\lambda=18 \mathrm{~cm})$} \\
\hline \multirow[t]{2}{*}{ J0854+2006 } & OJ 287 & Arecibo $300 \mathrm{~m}$ & 10 & 708 & 0.29 & 0.306 \\
\hline & & \multicolumn{5}{|c|}{ C-band $(\lambda=6 \mathrm{~cm})$} \\
\hline \multirow[t]{2}{*}{$\mathrm{J} 2202+4216$} & BL Lac & Effelsberg $100 \mathrm{~m}$ & 10 & 2124 & 0.10 & 0.0686 \\
\hline & & \multicolumn{5}{|c|}{ K-band $(\lambda=1.3 \mathrm{~cm})$} \\
\hline $\mathrm{J} 0750+1231$ & B $0748+126$ & GBT $100 \mathrm{~m}$ & 4.3 & 4215 & 0.05 & 0.889 \\
\hline
\end{tabular}

Column designation: (1) source name and its alias (2), (3) ground telescope, (4) and (5) - maximum baseline at which fringes were found, (6) angular scale, (6) redshift (MOJAVE database).

limit of a few $\times 10^{11} \mathrm{~K}$ [19]. These brightness temperature values may be reconciled with the standard $e^{-} / e^{+}$incoherent synchrotron radiation model if the emission is Doppler-boosted by a factor of $\delta \equiv[\Gamma(1-\beta \cos \theta)]^{-1} \sim 100$, where $\Gamma$ is the bulk Lorentz factor, $\beta$ the velocity in units of $c$ of the emitting plasma, and $\theta$ is the angle between the plasma flow direction and the line of sight. The large values of $\delta$ derived from the RadioAstron $T_{b}$ measurements combined with the equipartition inverse Compton limit argument are inconsistent with the typical values of $\delta$ derived from groundbased VLBI kinematic data [20,21]. This inconsistency might indicate that the jet flow speed is often higher than the jet pattern speed. More observations at long baselines are planned to probe the range of $T_{b} \sim 10^{14-15} \mathrm{~K}$. Surprisingly, interstellar scattering is not preventing fringe detection at long baselines even at $18 \mathrm{~cm}$.

\section{Acknowledgements}

The RadioAstron Space Radio Telescope was build and is operated by Lavochkin Association and Astro Space Center in collaboration with Russian and international institutions. The RadioAstron AGN Early Science Working Group is deeply grateful to the observers and technicians at Arecibo, ATCA, Effelsberg, Evpatoria, GBT, Hartebeesthoek, Hobart, Jodrell Bank, Medicina, Noto, Mopra, Parkes, Shanghai, Urumqi, Svetloe, Zelenchukskaya, Badary, Torun, Tidbinbilla, Usuda, WSRT, Yebes, and Robledo observatories for making this project possible.

This research was supported by the Russian Foundation for Basic Research (projects 11-0200368 and 12-02-33101), the basic research program "Active processes in galactic and extragalactic objects" of the Physical Sciences Division of the Russian Academy of Sciences, and the Ministry of Education and Science of the Russian Federation (agreement No. 8405).

\section{References}

[1] Gurvits, L., S. V. Pogrebenko, G. Cimo, D. Duev, G. Molera Calves, and T. Bocanegra Bahamon Planetary Radio Interferometry and Doppler Experiment (PRIDE): a multidisciplinary enhancement of space science missions, 39th COSPAR Scientific Assembly. Held 14-22 July 2012, in Mysore, India. Abstract C2.2-30-12, p.696, 39, (2012) 696 
[2] Henri, G. and L. Saugé The Bulk Lorentz Factor Crisis of TeV Blazars: Evidence for an Inhomogeneous Pileup Energy Distribution?, ApJ, 640, (2006) 185

[3] Kellermann, K. I. Brightness Temperature Constraints to Compact Synchrotron Source Radiation Obtained from IDV and VLBI Observations, PASA, 19, (2002) 77

[4] Beasley, A. J., D. Gordon, A. B. Peck, L. Petrov, D. S. MacMillan, E. B. Fomalont, and C. Ma The VLBA Calibrator Survey-VCS1, ApJS, 141, (2002) 13

[5] Fomalont, E. B., L. Petrov, D. S. MacMillan, D. Gordon, and C. Ma The Second VLBA Calibrator Survey: VCS2, AJ, 126, (2003) 2562

[6] Petrov, L., Y. Y. Kovalev, E. Fomalont, and D. Gordon The Third VLBA Calibrator Survey: VCS3, AJ, 129, (2005) 1163

[7] Petrov, L., Y. Y. Kovalev, E. B. Fomalont, and D. Gordon The Fourth VLBA Calibrator Survey: VCS4, AJ, 131, (2006) 1872

[8] Kovalev, Y. Y., L. Petrov, E. B. Fomalont, and D. Gordon The Fifth VLBA Calibrator Survey: VCS5, AJ, 133, (2007) 1236

[9] Petrov, L., Y. Y. Kovalev, E. B. Fomalont, and D. Gordon The Sixth VLBA Calibrator Survey: VCS6, AJ, 136, (2008) 580

[10] Fey, A. L., A. W. Clegg, and E. B. Fomalont VLBA Observations of Radio Reference Frame Sources. I., ApJS, 105, (1996) 299

[11] Fey, A. L. and P. Charlot VLBA Observations of Radio Reference Frame Sources. II. Astrometric Suitability Based on Observed Structure, ApJS, 111, (1997) 95

[12] Petrov, L., D. Gordon, J. Gipson, D. MacMillan, C. Ma, E. Fomalont, R. C. Walker, and C. Carabajal Precise geodesy with the Very Long Baseline Array, Journal of Geodesy, 83, (2009) 859

[13] Pushkarev, A. B. and Y. Y. Kovalev Single-epoch VLBI imaging study of bright active galactic nuclei at $2 \mathrm{GHz}$ and $8 \mathrm{GHz}, \mathrm{A} \& \mathrm{~A}, \mathbf{5 4 4},(2012) \mathrm{A} 34$

[14] Lee, S.-S., A. P. Lobanov, T. P. Krichbaum, A. Witzel, A. Zensus, M. Bremer, A. Greve, and M. Grewing a Global 86 GHz VLBI Survey of Compact Radio Sources, AJ, 136, (2008) 159

[15] Scott, W. K., and 19 colleagues The VSOP 5 GHz Active Galactic Nucleus Survey. III. Imaging Results for the First 102 Sources, ApJS, 155, (2004) 33

[16] Horiuchi, S., and 11 colleagues The VSOP 5 GHz Active Galactic Nucleus Survey. IV. The Angular Size/Brightness Temperature Distribution, ApJ, 616, (2004) 110

[17] Dodson, R., and 23 colleagues The VSOP 5 GHz Active Galactic Nucleus Survey. V. Imaging Results for the Remaining 140 Sources, ApJS, 175, (2008) 314

[18] Lovell, J. E. J., and 10 colleagues The Micro-Arcsecond Scintillation-Induced Variability (MASIV) Survey. II. The First Four Epochs, ApJ, 689, (2008) 108

[19] Readhead, A. C. S. Equipartition brightness temperature and the inverse Compton catastrophe, ApJ, 426, (1994) 51

[20] Cohen, M. H., M. L. Lister, D. C. Homan, M. Kadler, K. I. Kellermann, Y. Y. Kovalev, and R. C. Vermeulen Relativistic Beaming and the Intrinsic Properties of Extragalactic Radio Jets, ApJ, 658, (2007) 232

[21] Lister, M. L., M. H. Cohen, D. C. Homan, M. Kadler, K. I. Kellermann, Y. Y. Kovalev, E. Ros, T. Savolainen, and J. A. Zensus MOJAVE: Monitoring of Jets in Active Galactic Nuclei with VLBA Experiments. VI. Kinematics Analysis of a Complete Sample of Blazar Jets, AJ, 138, (2009) 1874 